Cochrane Schweiz, Institut universitaire de médecine sociale et préventive, Lausanne'; Service d'angiologie et d'Hémostase, Département des spécialités de médecine, Hôpitaux Universitaires de Genève ${ }^{2}$

'Theresa Bengough, ${ }^{2}$ Marc Righini, 'Erik von Elm

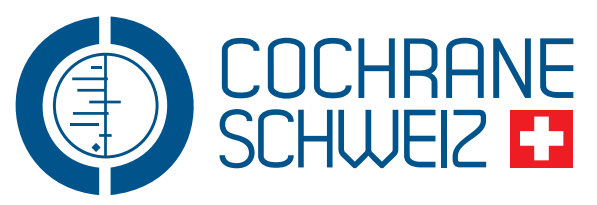

\title{
Thrombolyse bei tiefer Venenthrombose?
}

\section{Fallvignette}

Sie sehen eine 45-jährige Patientin mit einem bläulich-zyanotischen rechten Bein. Vor fünf Tagen habe ein Spannungsschmerz in der Wade begonnen und sich zunehmend in den Oberschenkel hochgezogen. Die rechte Wade misst im Umfang $4 \mathrm{~cm}$ mehr als die linke. Die Anamnese ergibt keine familiäre oder persönliche Vorgeschichte einer thromboembolischen Erkrankung. Die Patientin ist übergewichtig (BMI $35 \mathrm{~kg} / \mathrm{m}^{2}$ ) und nimmt seit einigen Jahren eine Östrogen-Progesteron-Kombination. Der Ultraschall zeigt eine Thrombose der tiefen Beinvenen, die bis in die äussere Beckenvene reicht.

\section{Frage}

Soll die Patientin nur mit Gerinnungshemmern oder zusätzlich mit einer Thrombolyse behandelt werden?

\section{Hintergrund}

Bei der tiefen Venenthrombose (TVT) führt die lokalisierte Blutgerinnung in einer Beinvene zur Bildung eines Thrombus; als Komplikation können Lungenembolie oder ein postthrombotisches Syndrom auftreten. Letzteres geht mit Schwellungen und Ödemen bis hin zum chronischen Unterschenkelgeschwür einher und tritt trotz Behandlung bei 30-50\% der Patienten auf. Die Standardtherapie mit Gerinnungshemmern soll eine Lungenembolie sowie ein
Wiederauftreten der TVT verhindern. Der aktualisierte Review verglich die Wirksamkeit dieser Standardtherapie mit der Kombination von Gerinnungshemmern und zusätzlicher Thrombolyse mit Streptokinase, Urokinase oder einem gewebespezifischen Plasminogen-Aktivator.

\section{Resultate}

Es wurden 17 randomisierte Studien eingeschlossen, an denen 1103 Erwachsene teilnahmen. Fünfzehn Studien untersuchten die systemische intravenöse Thrombolyse und zwei die Katheter-Lyse. Streptokinase war der am häufigsten verwendete Wirkstoff (10 Studien), jedoch waren die Dosierungsschemata nicht einheitlich.

Acht Studien ( $n=616)$ untersuchten die vollständige Thrombusauflösung. Diese wurde mit der Thrombolyse deutlich häufiger erreicht (RR 4,91; 95\% CI 1,66 bis 14,53). Ein signifikanter Effekt zeigte sich ebenfalls in der Verbesserung der Durchgängigkeit des betroffenen Venensegments (RR 2,48; 95\% CI 1,35 bis 4,57; 9 Studien, $n=440$ ). Signifikant weniger Patienten litten nach der Thrombolyse am postthrombotischen Syndrom (RR 0,64; $95 \%$ CI 0,52 bis 0,79; 4 Studien, $\mathrm{n}=352)$. Um dieses Therapieziel bei einem Patienten zu erreichen, mussten fünf Patienten behandelt werden $(\mathrm{NNT}=5)$. Die Katheter-Lyse senkte in einer der vier Studien (CAVENT) das Risiko eines postthrombotischen Syndroms nach 24 Monaten (RR 0,74; 95\% CI 0,55 bis 1,$00 ; n=189$ ). In der Metaanalyse aller 17 Studien zeigte sich ein signifikant erhöhtes Blutungsrisiko mit Thrombolyse (RR 2,23; 95\% CI 1,41 bis 3,52; $n=1103)$. Die Katheter-Lyse schien das Blutungsrisiko noch deutlicher zu erhöhen (RR 7,69; 95\% CI 0,40 bis 146,90; 2 Studien, $n=224$ ). Da nur drei Ereignisse auftraten, ist der Effekt jedoch statistisch nicht signifikant. Daten zur Frühmortalität (bis zu einem Monat) wurden in neun Studien erhoben $(\mathrm{n}=541)$, Daten zur Mortalität innerhalb sechs Jahren in drei Studien $(\mathrm{n}=344)$. Die Ergebnisse hierzu waren jedoch ebenfalls statistisch nicht signifikant. Auch zum Auftreten von Schlaganfällen lässt die Metaanalyse keine eindeutige Aussage zu, da nur drei Fälle (alle nach Thrombolyse) registriert wurden (RR 1,92; 95\% CI 0,34 bis 10,86).

\section{Einschränkungen}

- Die Datenlage war nicht ausreichend, um einen aussagekräftigen Vergleich unterschiedlicher Wirkstoffe oder Thrombolyse-Methoden anzustellen.

- Die Studien waren bezüglich der von der TVT betroffenen Beinvenensegmente sehr heterogen. Das ist relevant, da die Wahrscheinlichkeit von Komplikationen bei distalen Thromben als geringer gilt.

Im Artikel verwendete Abkürzungen: $95 \% \mathrm{Cl}$ Konfidenzintervall von $95 \%$

BMI Body Mass Index

NNT Number needed to treat (Anzahl der notwendigen Behandlungen)

RR Relatives Risiko

TVT Tiefe Venenthrombose 
- Daten zu anderen Nebenwirkungen wie allergischen oder anaphylaktischen Reaktionen wurden nicht erhoben. Es wurden auch keine Studien gefunden, die alleinige Thrombolyse und Therapie mit niedermolekularem Heparin miteinander verglichen.

\section{Schlussfolgerungen der Autoren}

Die Thrombolyse ist eine wirksame Behandlung bei der tiefen Venenthrombose. Sie erhöht die Venendurchgängigkeit, löst den Thrombus auf und reduziert das Auftreten postthrombotischer Syndrome. Wegen des erhöhten Blutungsrisikos empfehlen die Autoren jedoch strikte Auswahlkriterien. Dies schränkt die Anwendbarkeit dieser Behandlung ein. Obwohl die Resultate der beiden Thrombolyse-Methoden ähnlich sind, wird momentan die Katheter-Lyse der systemischen intravenösen Thrombolyse vorgezogen.

\section{Antwort auf die Frage}

Eine Thrombolyse wäre nur angezeigt, wenn die Patientin eine TVT der iliofemoralen Vene mit massiven Symptomen und dazu ein geringes Blutungsrisiko hätte. Da beides nicht zutrifft, ist eine konventionelle Therapie mit niedermolekularem Heparin (bzw. später Vitamin-K-Antagonist) und Kompressionsverband ausreichend. Das Östrogen-Progesteron-Präparat sollte zugunsten einer Verhütungsmethode ersetzt werden, die das Thromboserisiko nicht zusätzlich erhöht.

In der ATTRACT-Studie wird derzeit untersucht, ob durch Kombination der Katheter-Lyse mit einer mechanischen Thrombenentfernung die Wirkstoffdosis und damit das Blutungsrisiko verringert werden kann. Ergebnisse werden allerdings erst für 2016 erwartet (Clinicaltrials.gov NCT00790335).

\section{Korrespondenzadresse}

Dr. med. Erik von Elm

Cochrane Schweiz

Institut universitaire de médecine sociale et préventive

Corniche 10

1010 Lausanne

swiss.cochrane@chuv.ch

\section{Bibliographie}

Watson L, Broderick C, Armon MP: Thrombolysis for acute deep vein thrombosis. Cochrane Database Syst Rev 2014; 1: CDoo2783.

Diese Rubrik gibt die Resultate eines systematischen Reviews wieder, die durch die Cochrane Collaboration in der Cochrane Library (www.thecochranelibrary.com) publiziert wurde. Der Artikel beschränkt sich auf eine klar umschriebene Fragestellung und fasst den aktuellen Kenntnisstand dazu zusammen. Es handelt sich deshalb nicht um eine Leitlinie (Guideline) zur Behandlung einer bestimmten Erkrankung. Die Autoren beziehen sich auf den Inhalt des systematischen Reviews und stellen dabei die Auswahl der Studien für den Review nicht infrage. 